\title{
Tapinarof Cream 1\% Once Daily for Plaque Psoriasis: Secondary Efficacy Outcomes from Two Pivotal Phase 3 Trials
}

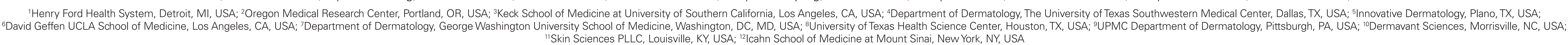

\section{SYNOPSIS}

Psoriasis is a chronic, immune-mediated disease characterized by scaly

erythematous, and pruritic plaques that can be painful, disfiguring, and sever

impact quality of life'

There is a need for efficacious and well-tolerated topical therapies for plaque psoriasis without restrictions on duration, site, and extent of use, or concerns with novel mechanisms have been US Food and Drug Administration (FDA)approved in over 20 years

Tapinarof is a first-in-class, non-steroidal, topical therapeutic aryl hydrocarbon eceptor modulating agent (TAMA) in development for the treatment of psoriasis and atopic dermatitis

- PSOARING 1 (NCT03956355) and PSOARING 2 (NCT03983980) were two pivotal phase 3 trials designed to assess the efficacy and safety of tapinarof cream $1 \%$ once dally (OD) in patients with mild to-severe plaque psoriasis

Primary efficacy endpoints and safety results from the two pivotal trials have been previously reported, demonstrating highly statistically significant efficacy

\section{OBJECTIVE}

To present the secondary efficacy endpoints in two pivotal phase 3 trials of

tapinarof cream 1\% QD for the treatment of plaque psoriasis

\section{METHODS}

\section{Study Design}

In two identically designed, phase 3, multicenter (US and Canada), double-

blind, vehicle-controled randomized trials, patients with mild-to-severe plaque

12 weeks (Figure 1 )

- Following the double-blind period, patients could enroll in an open-label, longerm extension trial or complete a follow-up visit 4 weeks after the end of

treatment (Week 16)

Figure 1. Study Design

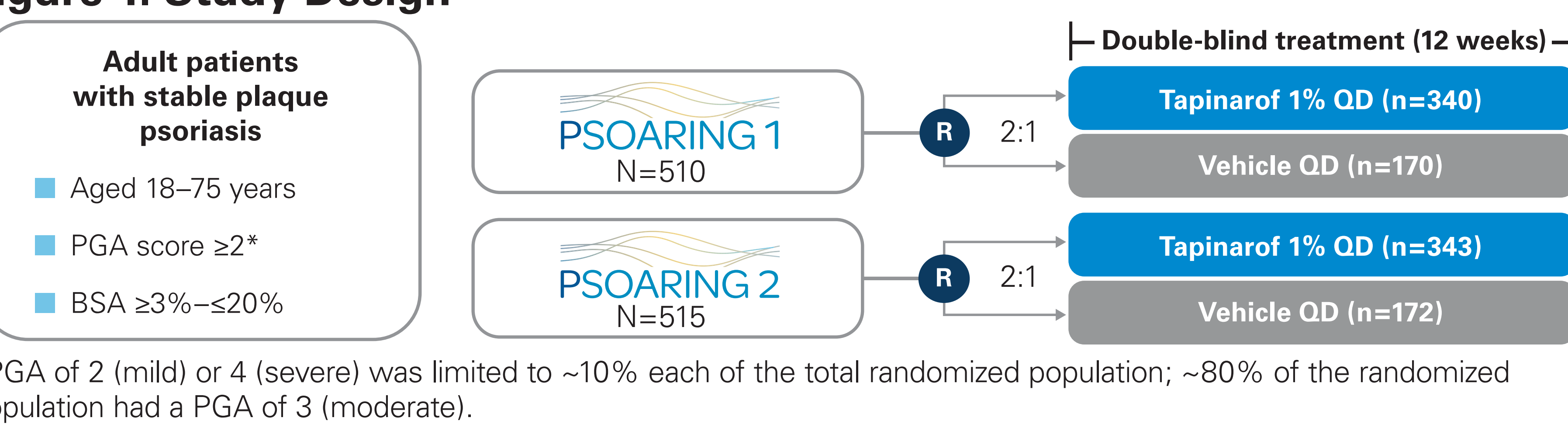

\section{Endpoints and Statistical Analysis}

The primary efficacy endpoint was Physician Global Assessment (PGA)

response at Week 12, defined as the proportion of patients with a PGA score

of clear (0) or almost clear (1) and $\geq 2$-grade improvement in PGA score from

baseline to Week

Secondary and exploratory endpoints included the following

Proportion of patients with $\geq 75 \%$ improvement in Psoriasis Area and Severity

Index (PASI75) score from baseline at Week 12

Proportion of patients with a PGA score of clear (0) or almost clear (1) at

Week 12

Mean change in percentage body surface area (\%BSA) affected from

baseline to Week 12

Proportion of patients with $\geq 90 \%$ improvement in Psoriasis Area and Severity Index (PASI90) score from baseline at Week 12

Proportion of patients with a PASI75, PGA score of clear (0) or almost clear (1), or PASI90; and the mean change in \%BSA affected from baseline at

The incidence, frequency, and nature of adverse events (AEs) and serious AEs were monitored from the start of study treatment until the end-of-study visit Efficacy endpoints were derived from the intention-to-treat (ITT) population using multiple imputation analysis for missing data

For categorical endpoints, $P$ values for differences between tapinarof cream and vehicle in both trials were calculated using Cochran-Mantel-Haenszel analysis and stratified by baseline PGA score. $P$ values for continuous variables were analyzed using analysis of covariance, with randomized treatment effect is presented as least squares mean

\section{RESULTS}

Patient Disposition and Baseline Characteristics

In PSOARING 1 and 2, a total of 510 and 515 patients were randomized (ITT

population), respectively, across 97 sites in the US and Canada

treatment groups and trials (Table
At baseline, $79.2 \%$ and $83.9 \%$ of patients had a PGA score of 3 , mean (standard deviation [SD]) PASI score was 8.9 (4.1) and 9.1 (3.8), and mean (SD) \%BSA affected was 7.9 (4.8) and 76 (4.3) in PSOARING 1 and 2, respectively Table 1. Baseline Patient Demographics and Disease Characteristics

\begin{tabular}{|c|c|c|c|c|}
\hline & \multicolumn{2}{|c|}{ PSOARING 1} & \multicolumn{2}{|c|}{ PSOARING 2} \\
\hline & $\begin{array}{c}\text { Tapinarof } 1 \% \\
\text { OD } \\
(n=340)\end{array}$ & $\begin{array}{l}\text { Vehicle QD } \\
(n=170)\end{array}$ & $\begin{array}{c}\text { Tapinarof } 1 \% \\
\text { OD } \\
(n=343)\end{array}$ & $\begin{array}{c}\text { Vehicle QD } \\
(\mathrm{n}=172)\end{array}$ \\
\hline Mean age, years (SD) & $49.8(13.7)$ & $49.1(13.3)$ & $50.0(13.1)$ & $50.0(13.7)$ \\
\hline Male, $\mathbf{n}(\%)$ & $213(62.6)$ & $86(50.6)$ & $188(54.8)$ & $102(59.3)$ \\
\hline Weight, kg, mean (SD) & $91.7(24.6)$ & $92.8(22.7)$ & $92.9(24.3)$ & 89.6 (19.9) \\
\hline BMI, kg/m², mean (SD) & $31.4(7.8)$ & 32.5 (7.6) & 31.8 (7.7) & $30.7(6.3)$ \\
\hline \multicolumn{5}{|l|}{ PGA, $n(\%)$} \\
\hline 2 - Mild & $39(11.5)$ & $21(12.4)$ & $28(8.2)$ & $15(8.7)$ \\
\hline 3 - Moderate & $271(79.7)$ & $133(78.2)$ & $288(84.0)$ & $144(83.7)$ \\
\hline 4- Severe & $30(8.8)$ & $16(9.4)$ & 27 (7.9) & $13(7.6)$ \\
\hline PASI, mean (SD) & $8.7(4.0)$ & $9.2(4.4)$ & $9.1(3.7)$ & $9.3(4.0)$ \\
\hline BSA affected, $\%$, mean (SD) & $7.8(4.6)$ & $8.2(5.1)$ & $7.8(4.4)$ & $7.3(4.1)$ \\
\hline
\end{tabular}

\section{Primary Endpoint: PGA Response ${ }^{2}$}

As previously reported, PGA response rates were highly statistically significant in the tapinarof cream $1 \%$ OD group versus the vehicle group in both PSOARING 1 and $2: 35.4 \%$ vs $6.0 \%$ and $40.2 \%$ vs $6.3 \%$ (both $P<0.0001$ )

PASI75 Response Rate from Baseline to Weeks 2, 4, 8, and 12

Significance in PASI75 response was demonstrated as early as Week 4 in both PSOARING $1(P=0.0030)$ and $2(P=0.0002)$, with a significantly higher mean proportion of patients in the tapinarof group than the vehicle group achieving $P<0.0001$ ), respectively (Figure 2)

Figure 2. PASI75 Response Rates from Baseline to Weeks 2, 4, 8, and 12

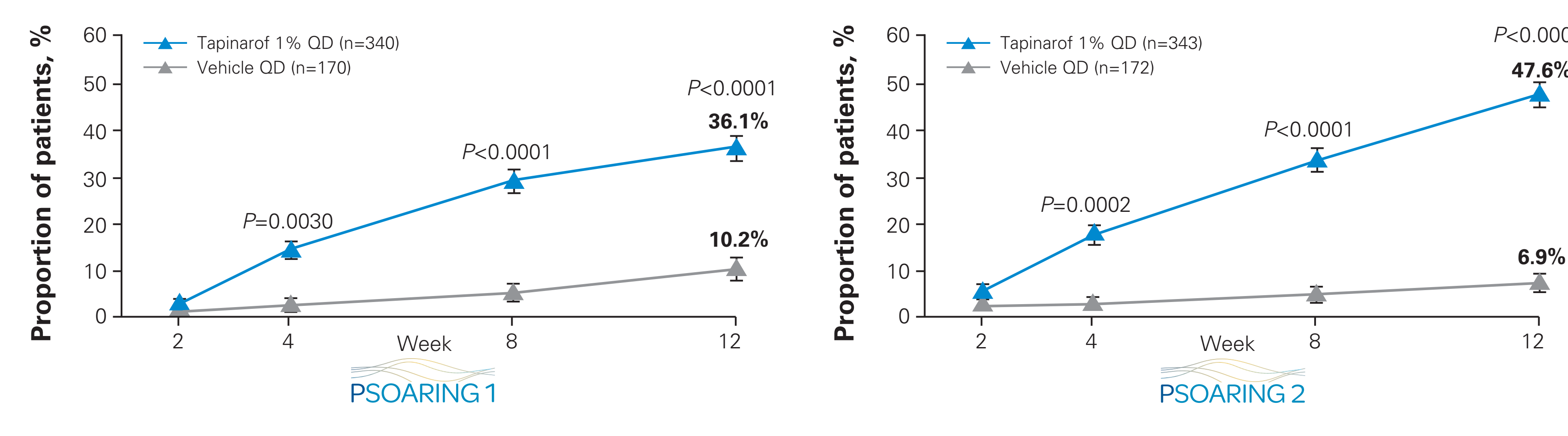

Area and Severity Index; $Q D$, once daily; SE, standard error. Significance in achievement of PGA score of 0 (clear) or 1 (almost clear) was demonstrated as early as Week 4 in PSOARING $1(P=0.0069)$ and Week 2
in PSOARING $2(P=0.0389)$, with a significantly higher mean proportion of patients in the tapinarof group than the vehicle group achieving a PGA score of respectively (Figure 3 )

Figure 3. PGA Score of 0 or 1 at Weeks 2, 4, 8, and 12

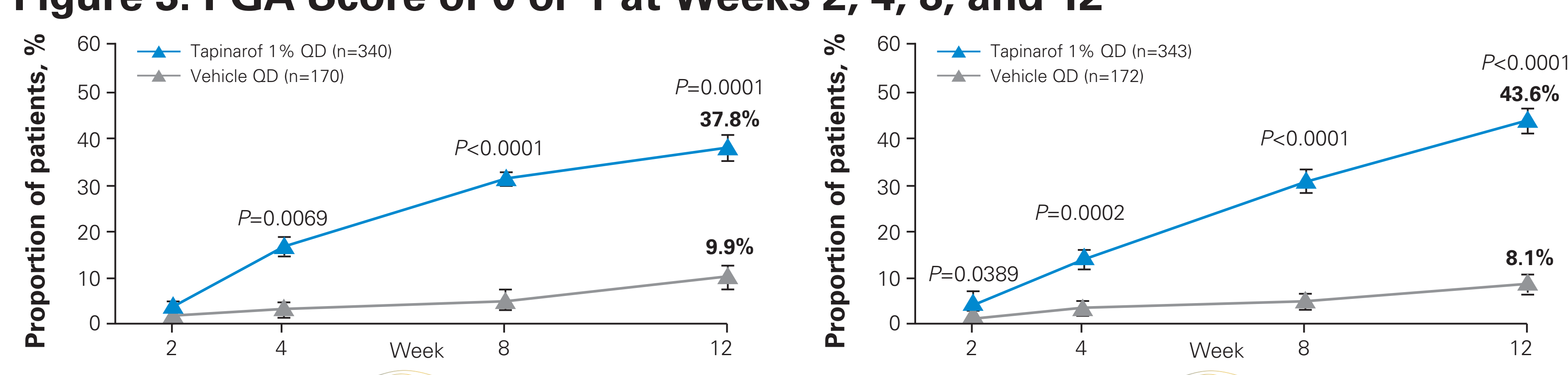

. once daily, SE, standard error.
.

Mean Change in \%BSA Affected from Baseline to Weeks 2, 4, 8, and 12 significant improvements from Week $2(P \leq 0.0027)$ reaching -3.5 vs -0.2 and -4.2 vs 0.1 at Week 12 in PSOARING 1 and 2, respectively $(P<0.0001$ in both trials) (Figure 4)

Figure 4. Change in \%BSA Affected from Baseline to Weeks 2, 4, 8, and 12

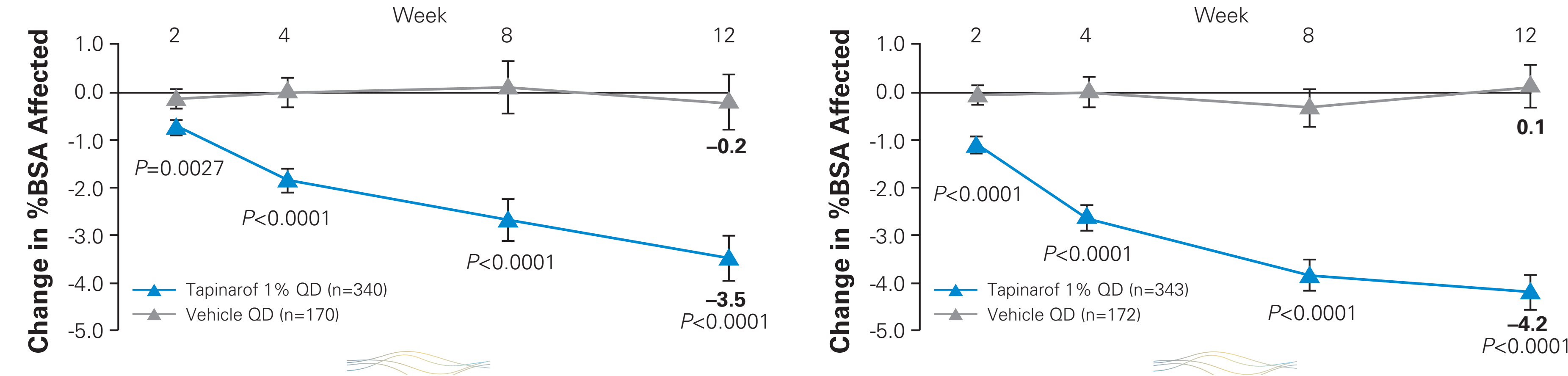

PASI90 Response Rate from Baseline to Weeks 2, 4, 8, and 12

Significance in PASI90 response was demonstrated as early as Week 8 in both proportion of patients in the tapinarof group than the vehicle group achieving

\section{PASI90 response at Week 12: $18.8 \%$}

Figure 5. PASI90 Response Rate from Baseline to Weeks 2, 4, 8, and 12

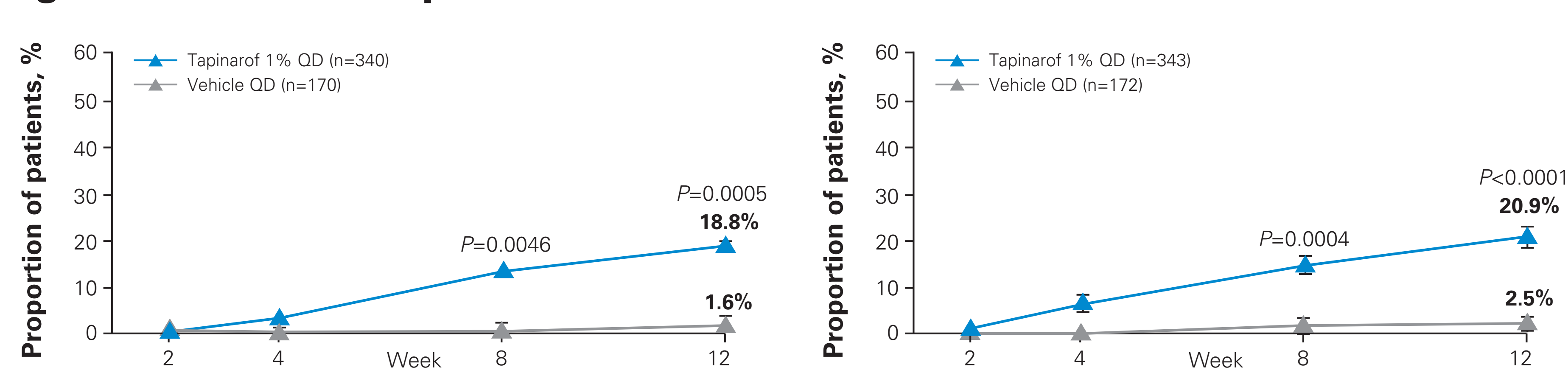

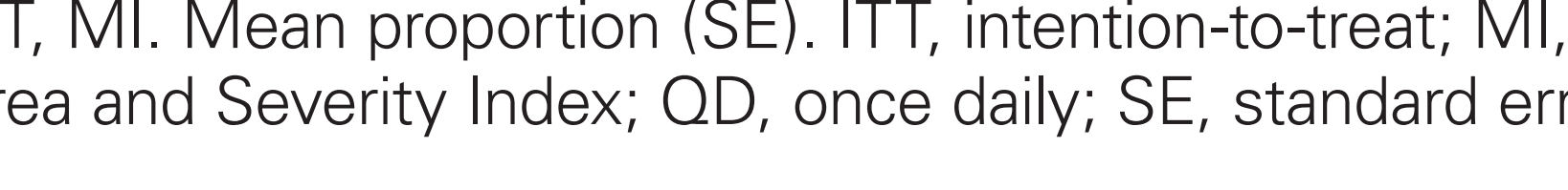

Figure 6 displays photographs of the clinical response of a patient treated with tapinarof cream who achieved the primary and secondary efficacy endpoints

Figure 6. Clinical Response of a Patient with Plaque Psoriasis who Achieved Primary and Secondary Efficacy Endpoints

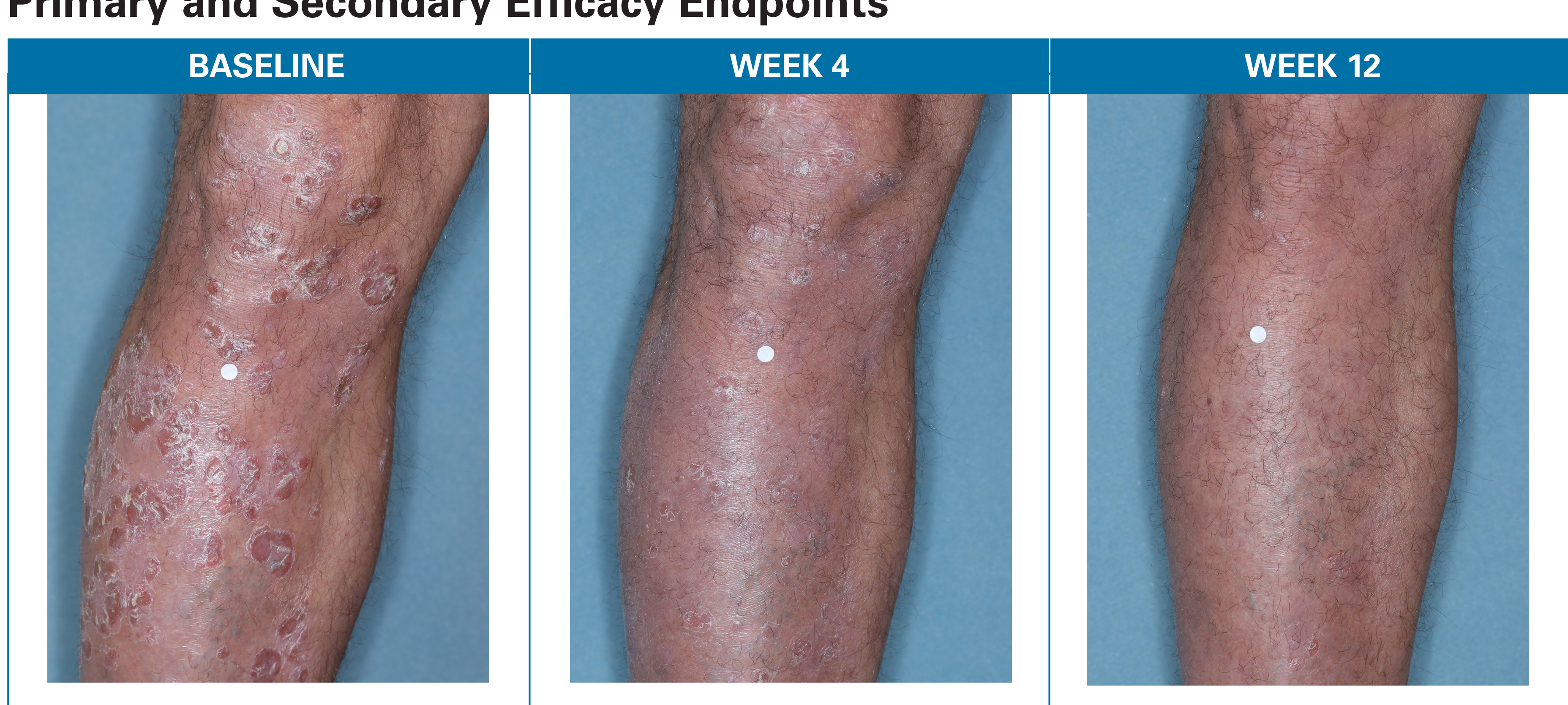

PGA and PASI

As previously reported, ${ }^{2}$ most treatment-emergent AEs (TEAEs) in PSOARING

and 2 were mild or moderate in severity, consistent with previous studies, ${ }^{3,4}$

and most did not lead to study discontinuation

The most common ( $\geq 1 \%$ in any group) treatment-related TEAEs were folliculitis, ontact dermatitis, headache, pruritus, and dermatitis

Folliculitis was mostly mild or moderate in severity in both studies, and study discontinuation due to folliculitis was low in PSOARING 1 and $2: 1.8 \%$
$(6 / 340)$ vs $0.0 \%(0 / 170)$ and $0.9 \%(3 / 343)$ vs $0.0 \%(0 / 172)$, respectively

\section{CONCLUSIONS}

Tapinarof cream 1\% QD significantly improved all measures of disease activity and showed rapid, clear, and consistent separation versus vehicle as early as the irst clinical assessment at Week 2

- These findings are consistent with the superior clinical efficacy and good tolerability profile of tapinarof cream reported previously $y^{24}$

Early improvements continued throughout the trials and did not reach maxima effect by Week 12, as confirmed by results from a long-term extension trial ${ }^{5}$ Tapinarof cream 1\% OD has the potential to be the first topical, non-steroidal psoriasis treatment with a novel mechanism of action in over 20 years

\section{REFERENCES}

Menter A, et al. J Am Acad Dermatol. 2008:58:826-850; 2. Lebwohl M, et al. N Engl J Med.
J 221;385:2219-2229; 3. Robbins K, et al. J Am Acad Dermatol. 2019;80:714-721; 4. Stein Gold L, et
J Am Acad Dermatol. 2021;84:624-631; 5. Strober B, et al. Innovations in Dermatology Virtual Spring

\section{ACKNOWLEDGMENTS}

The authors thank the participating investigators, patients and their families, and colleagues involved in the of educational presentations, and/or has received grants from Arcutis, Amgen, Bristol-Myers Squibb, as a scientific adviser and/ or clinical study investigator for AbbVie, Abcentra, Aligos, Almirall, Amgen, Arcults,

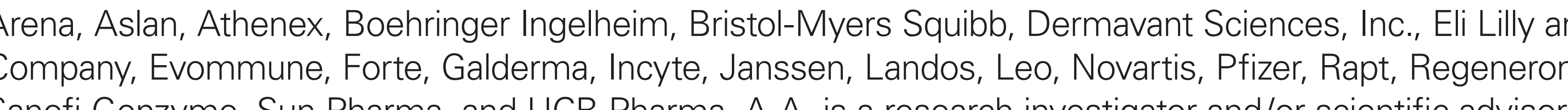
AbbVie, Bristol-1yyers Squibbb, Boehringer Ingelheim, EPI, Incyte, Leo, UCB, Janssen, Lilly, Novartis, Ortho
Dermatologios. Sur, Dermant Sciences Inc., Dermira, Sanofi, Regeneron, Pfizer, and Modmed. S.R.D.

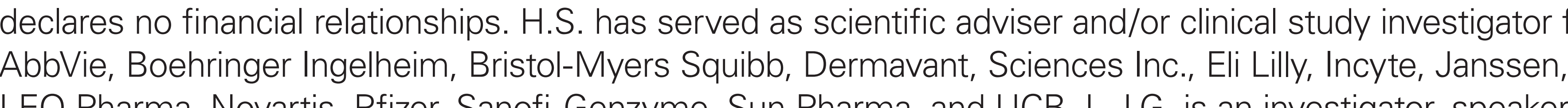

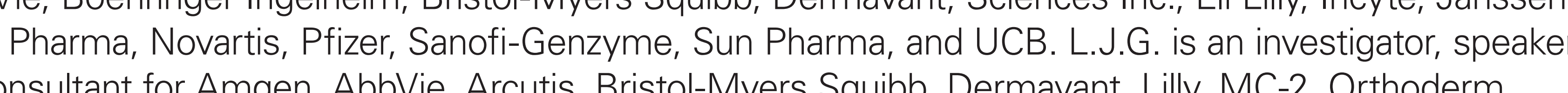
L.K.F. is an investigator for AbbVVie, Amgen, Arcutis, Bristol-Myers Squibb, Boehringer Ingectheim, Dermavant, Mli Lilly, Galderma, Janssen, Novartis, Regeneron and UCB, and a consultant for AbbVVie, Arcutis, Bristolguidance of the authors was provided by ApotheCom, UK, and was funded by Dermavant Sciences, Inc. In 